\title{
EFECTOS DEL USO DEL ANDADOR SOBRE EL DESARROLLO PSICOMOTOR Y RAZONES PARA SU USO
}

Effects from using babywalkers on the psychomotor development and the reasons for its use

Efeitos do uso do andador para o desenvolvimento e a razões para seu uso

\section{Dra. Verónica Delgado Mayorga}

Centro de Rehabilitación Infantil CRIAS, Viña del Mar, Chile. Telf.: 56-32-2693562. Correo electrónico: crias_chile@hotmail.com

\section{Resumen}

El andador aún se usa con frecuencia, si bien en el ámbito médico existe el convencimiento de que no sólo NO es útil, sino que interfiere con el Desarrollo Psicomotor (DSM), así como favorece las caídas y traumatismos en el niño.

Objetivo: analizar los motivos para usarlo y sus efectos sobre el DSM, aplicando una encuesta a 168 padres de niños normales menores de 24 meses, que ya lograron la marcha.

Resultados: el 63,1\% usó andador, 77,4\% menos de 3 horas diarias y 8,5\% más de 6 horas. Motivos para usarlo fueron "por comodidad" $(36,8 \%)$ y "para que caminara" (34,9\%). 65,4\% de los niños que asistían a Jardín Infantil usaron andador, versus el 61,9\% de los que no asistían, siendo en algunos su uso precisamente en el Jardín. El uso es mayor en los varones (66,9\% vs 55,9\%). En relación a los efectos, $80,4 \%$ de los niños que no gatearon, usaron andador; la marcha en equino y los choques con muebles o paredes, fueron más frecuentes en los niños que usaron andador $(87,7 \%$ y $74,6 \%$ respectivamente) y en relación a las reacciones protectoras de brazos, fueron deficientes en el 43,4\% de los niños que usaron andador, versus el 12,9\% de aquellos que no usaron. La edad de gateo, bipedestación y marcha fue $1-2$ meses mayor en los niños que usaron andador.

Conclusión: el andador no sólo no apoya el DSM sino que potencia deficiencias del mismo.

Palabras clave: andador, desarrollo psicomotor, gateo, marcha equino. 


\begin{abstract}
Baby walker (BW) is an instrument that is still used frequently. However, in medical field there is the conviction that it is not only NOT useful, but also interferes the Psychomotor Development, as well as cause falls and injuries in the child.

Objective: to analyse the reasons for using BW and those effects, applying a survey to 168 parents of normal children under 24 month, that had achieved independent walking.

Results: $63.1 \%$ used BW, 77,4\% under 3 hours daily and 8,5\% more than 6 hours per day. Reasons were "for convenience" (36.8\%) and "stimulate walking" (34.9\%). From the children attending Kindergarten, $65.4 \%$ used babywalkers, in front of $61.9 \%$ of those who did not assist. Moreover, in some children, their use was done just in the Garden. The use was greater in males (66.9\% vs 55.9\%). Concerning their effects, children who did not crawl, $80.4 \%$ had used baby walkers; equine gait and shocks with furniture or walls were more frequent in children who used BW $(87.7 \%$ and $74.6 \%$ respectively) and in relation to arm protective reactions, in $43.4 \%$ of children who used BW they were poor, versus $12.9 \%$ of those who did not use. The age of crawling, standing and walking was 1 - 2 months later in children who used BW.
\end{abstract}

Conclusion: BW not only does not stimulate psychomotor development but also potentiates deficiencies

Key Words: baby walker, psychomotor development, crawling, equine gait.

\title{
Resumo
}

O andador ainda é usado com frequência, embora não haja na área médica estão convencidos de que não só não é útil, mas interfere com o desenvolvimento psicomotor (DSM) e favorece quedas e trauma para a criança.

Objetivo: Analisar as razões para o uso e seus efeitos sobre o DSM, usando uma pesquisa com 168 pais de crianças normais com menos de 24 meses, que já alcançaram progressos. 
Resultados: 63,1\% utilizam andador, $77,4 \%$ inferior a 3 horas por dia e $8,5 \%$ ao longo de 6 horas. Razões para o uso eram "por conveniência" (36,8\%) e "caminhar" $(34,9 \%) .65,4 \%$ das crianças que frequentam o Jardim de infancia usam andador, contra $61,9 \%$ daqueles que não frequentam, sendo que em alguns usam precisamente no Jardim de infancia. A utilização é superior nos meninos (66,9\% vs 55,9\%). Em relação aos efeitos, $80,4 \%$ das crianças que não engatinharam, usando andador; A marcha em equino e confrontos com móveis ou paredes, foram mais comuns em crianças que usaram andador $(87,7 \%$ e $74,6 \%$, respectivamente) e em relação às reações protetoras de braços eram deficientes em 43,4\% das crianças que usaram andador, contra 12,9\% daqueles que não foram utilizados. A idade de engatinhar, em ficar em pé e andar com 1 - 2 meses maior em crianças que usaram um andador.

Conclusão: o andador não só unicamente não apoia no DSM, sendo que pode causar deficiencia do mesmo.

Palavras-chave: andador, desenvolvimento psicomotor, rastejamento, marcha de equino.

\section{Introducción}

El uso del andador es una práctica aún común en nuestro medio y en muchos otros lugares del mundo. Su uso documentado se remonta a la Edad Media, encontrándose ilustraciones y modelos de aquella época en museos. Probablemente en sus inicios, el objetivo fuera proteger al niño de los peligros del suelo, desde infecciones, hasta lesiones por animales. Con el tiempo han ido evolucionando, encontrándose hoy en el mercado una gran variedad de modelos.

Existen en la literatura artículos que hablan de los problemas que genera el uso del andador, tanto desde el punto de vista de favorecer los traumatismos (1) como de alterar el proceso de adquisición de los hitos del Desarrollo Psicomotor (2). Sin embargo en nuestro medio hay escasas publicaciones al respecto, por lo que quisimos investigar tanto las causas de su uso, como sus efectos.

Dentro de los argumentos que se esgrimen actualmente para justificar el uso del andador están el que "fortalezca las piernas" para que pueda caminar mejor; dejarlo allí para poder hacer las cosas del hogar; entretención del niño; comodidad de los cuidadores. 
Sin embargo, los que trabajamos en salud y específicamente en rehabilitación infantil, nos damos cuenta que el uso del andador, no sólo no es beneficiosos, sino que junto con ser un "generador" de traumatismos del niño por las caídas del o con el andador, acarrea además una serie de consecuencias para el desarrollo del niño. Entre éstas, el que muchos niños que usan andador no gatean; o que al iniciar la marcha lo hacen en puntas de pie, se caen más frecuentemente o que chocan con los muebles o paredes. Dado que existe la creencia que el andador ayuda a la marcha, quisimos determinar también si los niños que lo utilizan, caminan antes o después que los que no lo hacen.

Por último, el Jardín Infantil es una instancia de estimulación para el niño, al interactuar con sus pares y participar en las actividades pedagógicas de ese nivel. Junto con ello, debiera ser también una instancia de educación a la familia en temas de desarrollo y crianza. Por ello nos interesaba saber si había diferencias de uso de andador entre los niños que asistían a Jardín y los que no lo hacían.

\section{Objetivo}

El objetivo de este estudio es conocer las causas que llevan al uso del andador, así como revisar sus efectos sobre algunos hitos del Desarrollo Psicomotor.

\section{Material y método}

Se aplicó una encuesta a padres de lactantes que asisten a control sano, que ya hubieran logrado la marcha independiente y que fueran menores de 24 meses, de diferentes centros de salud de la región de Valparaíso, sin patologías neuromotoras. El estudio se realizó en el marco de un trabajo de Tesis para la obtención del título de Kinesiología de la Universidad del Mar (3).

Se probó la validez de la encuesta consultando sobre su contenido a profesionales del área de salud, especialistas en el área del Desarrollo Psicomotor. Y la confiabilidad de la misma se probó re-encuestando aleatoriamente a 10 padres y/o cuidadores.

La Encuesta se dividió en 2 partes:

a) Aspectos generales del ambiente y/o cuidadores

a. Referente a uso/ no uso de andador, 

b. Horas de uso al día,
c. Motivos para su uso.
d. Tipo de domicilio, número de hermanos,
e. Asistencia o no a Jardín Infantil

b) Aspectos del niño, hitos del Desarrollo Psicomotor
a. Sexo
b. Logro del gateo o no
c. Marcha en puntas de pie
d. Choque con muebles, paredes o personas al caminar
e. Reacciones protectoras de brazos al caerse
f. Frecuencia de caídas al caminar
g. Edad de gateo, bipedestación y marcha

\section{Resultados}

La distribución por sexo de la muestra fue de 59 niñas $(35,12 \%)$ y 109 niños $(64,88 \%)$. De ellos, el 63,1\% usó andador, siendo la distribución por sexo de 55,9\% de las niñas y 66,9\% de los niños (Gráfico 1).

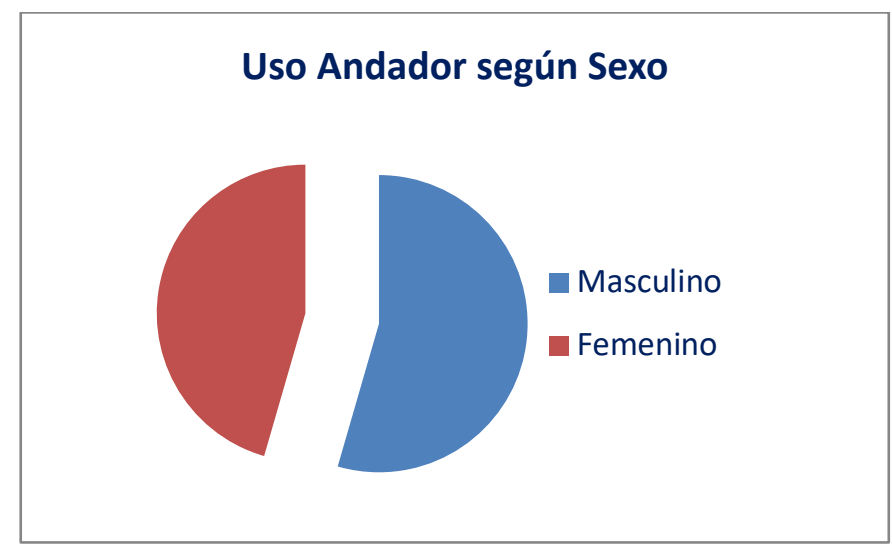

Gráfico 1: Uso Andador según Sexo 
El 32,7 \% de los niños asistió a Jardín, en jornada parcial o completa. De ellos, el 65,4\% utilizó andador, versus el 61,9 \% de los que habían estado sólo en el hogar, a cargo principalmente de sus madres.

Con respecto al número de horas de uso, el 77,4\% lo usó menos de 3 horas al día, $14,2 \%$ de 3-6 horas y un 8,5\% lo usó más de 6 horas.

De toda la muestra, el 69,6\% logró el hito del gateo. De los que no lo lograron, el 80,4\% utilizó andador. En el grupo de los que sí gatearon, sólo el 55,5\% había utilizado andador (Gráfico 2). De los niños que usaron andador, sólo el 61,3\% logró el gateo.
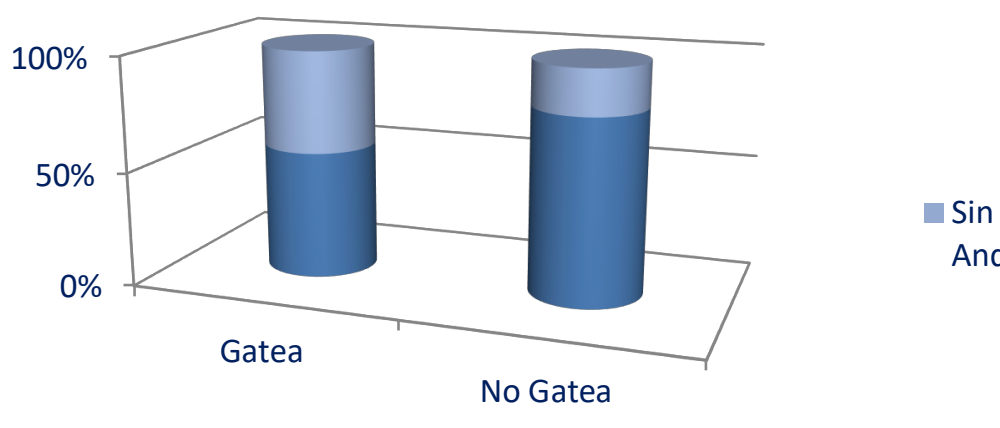

Andador

Gráfico 2: porcentaje de niños que gatean y los que no lo hacen en relación al uso de andador

Con respecto a la presencia de "postura en punta de los pies al caminar" $(38,7 \%$ del total), el $87,7 \%$ de los niños que la presentaron, había usado andador.

En relación al ítem "choca con las paredes, muebles o personas" al caminar (70,2\% del total), el 74,6\% había usado andador. 


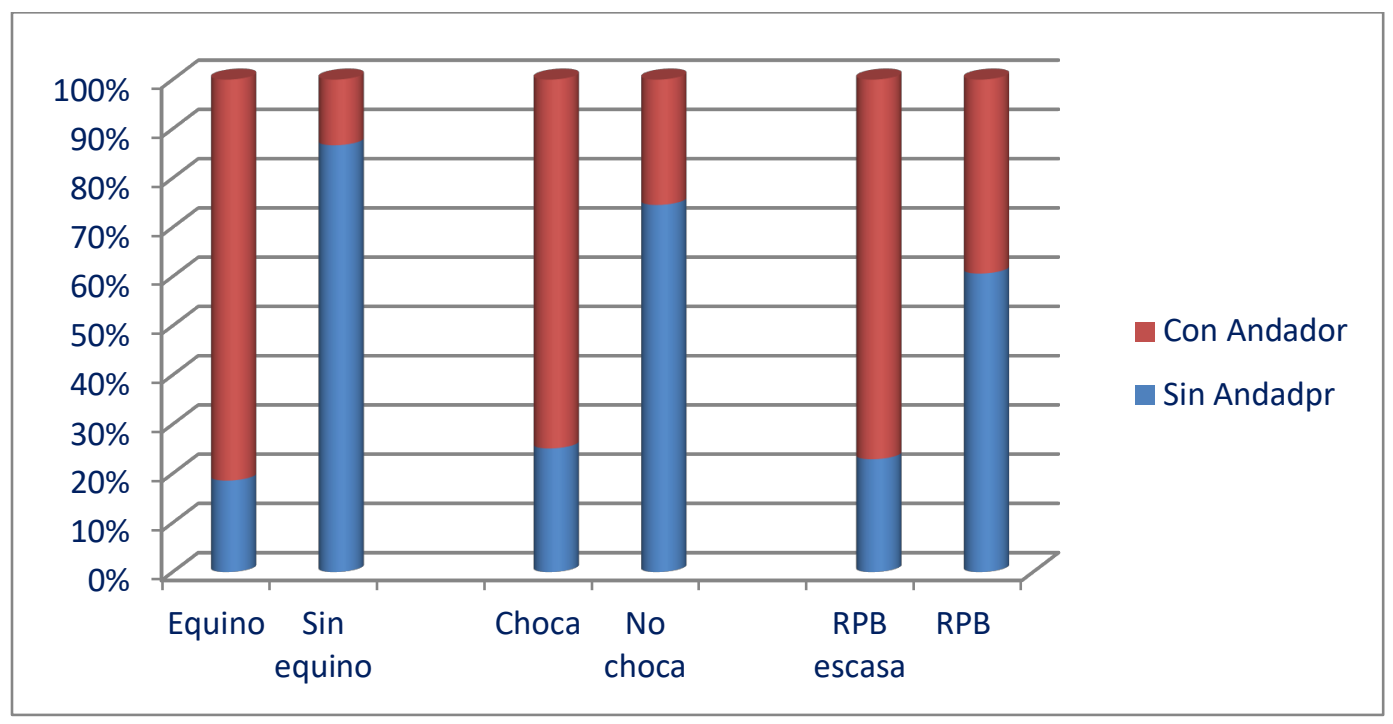

Gráfico 3: Comparación de niños que caminan en puntas de pie, que chocan con muebles o paredes y que tienen reacciones protectoras de los brazos deficientes en relación al uso de andador.

Con respecto al ítem "reacciones protectoras de los brazos al caerse" (RPB), es decir a la función de apoyo y protección de las manos o reacción de paracaídas anterior, se consultó en cuanto a si era una reacción habitual o se presentaba de manera escasa o ausente. Del total de la muestra, el 67,9\% de los niños tenía RPB adecuadas. Al comparar este ítem en relación al uso de andador se observó que el 43,4\% de los niños que usaron andador tuvieron RPB deficientes, versus sólo el 12,9\% de los niños que no lo usaron.

En relación a la edad de aparición de gateo, bipedestación y marcha independiente, se observó que en promedio, éstas aparecieron $1-2$ meses más tarde en los niños que habían utilizado andador (Tabla 1). 


\begin{tabular}{|lcc|}
\hline & $\begin{array}{c}\text { Sin } \\
\text { Andador }\end{array}$ & $\begin{array}{c}\text { Con } \\
\text { Andador }\end{array}$ \\
\hline Gateo & $8 \mathrm{~m}$ & $9 \mathrm{~m}$ \\
\hline Bipedestación & $10 \mathrm{~m}$ & $11 \mathrm{~m}$ \\
\hline Marcha libre & $12 \mathrm{~m}$ & $14 \mathrm{~m}$ \\
\hline
\end{tabular}

Tabla 1: Edad de gateo, bipedestación y marcha en niños que usaron andador y aquellos que no lo hicieron

\section{Discusión y Conclusiones}

La costumbre del uso del andador sigue siendo de alta prevalencia, con la idea de facilitar el cuidado a los padres o estimular al niño. Según los resultados obtenidos, especialmente en relación al desarrollo psicomotor, queda en evidencia que su uso no sólo no favorece el desarrollo, sino que potencia una mayor inestabilidad en la marcha, retrasando incluso la aparición de aquellos que justamente pretende estimular. Junto con ello, altera la formación del esquema corporal del niño, ya que al desplazarse dentro del andador, cambia de dirección cuando choca o topa con algo, por lo que "aprende" que cuando choca es cuando debe cambiar de dirección, patrón que se mantiene cuando inicia la marcha, al menos en los primeros meses.

El gateo es un hito cuya relevancia se suele poner en discusión ya que hay niños que no lo realizan, pero igualmente adquieren la marcha independiente. Sin embargo, este hito tiene relevancia no sólo desde el punto de vista neuromotor, requiriendo del funcionamiento óptimo del sistema nervioso central, sino también desde el punto de vista socio-emocional, cognitivo y sensorial (4). El uso del andador, especialmente si es de inicio precoz, se traduce en una menor incidencia de aparición de este hito, como se pudo comprobar en este estudio ( $80,4 \%$ de los niños que no gatearon, usaron andador). 
El Jardín infantil como se mencionaba antes, es una instancia de estimulación para los niños, pero debiera también ser una instancia de educación para los padres en cuanto a normas de crianza, manejo y estimulación de los niños. Por lo tanto, llama la atención que en cuanto al uso de andador, la asistencia al Jardín no fuera un factor protector, sino incluso se observó un discreto mayor uso de andador en quienes asistieron a él. Más aún, sorprende y preocupa que aún hoy, haya Jardines que cuentan con ellos, al igual que con otros elementos probablemente igual de nocivos como los centros de estimulación estáticos (andadores sin ruedas) o las "saltarinas".

En suma, en todos los aspectos evaluados, los niños que usaron andador mostraron resultados más deficientes. Por lo tanto, su uso no sólo no es recomendable, sino que debiera desaconsejarse fuertemente.

\section{Referencias bibliográficas}

Delgado, V. y Contreras, S. (2015). Desarrollo Psicomotor normal, primeros años, Ed Mediterráneo.

Diaz, J., Serey, J., Soto, J. y Tolosa, A. (2010).Influencia del uso del andador sobre el proceso de adquisición de la marcha independiente en niños menores de 24 meses. Seminario para optar al título de kinesiólogo y grado de licenciado en kinesiología, Universidad del Mar, 2010.

Santos, L. et al. (1996). Patrones de uso, creencias populares y accidentabilidad por andador infantil, bases para una campaña sanitaria. Anales españoles de Pediatría, 44, 4, 337-340.

Triviño, X., Bedregal, P., Azocar, M., Valenzuela, P. y González, C. (1997). Uso del andador. Rev. Chil. Pediatría 68, 6, 256-259. 